\title{
Chandra Study of the Central Object Associated with the Supernova Remnant MSH 11-62
}

\author{
Ilana Harrus \\ Goddard Space Flight Center, Greenbelt, MD 20771, USA \\ Joseph P. Bernstein \\ University of Michigan, Ann Arbor, MI 48109, USA \\ Patrick O. Slane, Bryan Gaensler \\ Harvard-Smithsonian Center for Astrophysics, Cambridge, MA 02138, \\ $U S A$ \\ John P. Hughes \\ Rutgers University, Piscataway, NJ 08854, USA \\ David Moffett \\ Furman University, Greenville, SC 29613, USA \\ Richard Dodson \\ Japan Aerospace Exploration Agency (JAXA), Kanagawa, 229-8510, \\ Japan
}

\begin{abstract}
We present results from our analysis of Chandra data on the supernova remnant MSH 11-62 (also known as G291.0-0.1). Our previous $A S C A$ analysis showed that MSH 11-62 is most likely a composite remnant whose strong non-thermal emission is powered by a compact object, most probably a pulsar. The present analysis confirms in a spectacular fashion the earlier detection of a compact source. The Chandra data reveal a small region with a hard non-thermal spectrum located at the tip of the central radio emission seen in data taken at the Australia Telescope Compact Array (ATCA). This source is likely the young rapidly rotating neutron star powering the synchrotron nebula in MSH 11-62. Compared to other young rotation-powered pulsars the Chandra spectrum of MSH 11-62 implies an energy loss rate of $\dot{E} \sim 5 \times 10^{36}$ ergs $\mathrm{s}^{-1}$.
\end{abstract}

\section{Introduction}

When the collapse of a massive star results in the formation of a neutron star, the canonical picture leads one to expect a pulsar accompanied by a shell-type 
remnant produced by the blast wave associated with the supernova explosion. In cases of unfavorable viewing angles relative to the pulsar spin axis, the pulsar may not be observed. Nevertheless, this central object (pulsating or not) powers, through rotational kinetic energy loss, a magnetic field bubble in which electrons are accelerated to relativistic energies. The synchrotron radiation emitted by these electrons is one sure sign of the presence of a compact object, even when no pulse is detected (i.e., the detection of the synchrotron nebula does not suffer from the known bias of favorable viewing angle mentioned earlier). X-ray observations of composite remnants are crucial because they provide such a direct way of identifying a central synchrotron component, thus giving unambiguous proof that the remnant was produced by the collapse of a massive star (i.e., a Type Ib or Type II SN); further studies of the synchrotron nebula (also called the "plerion" or the "pulsar wind nebula" or PWN) help restrict the parameter space for the characteristic values of the pulsar. In particular, one can estimate the pulsar's spin-down luminosity $\dot{E}$, and the initial spin period of the neutron star (see, for example, Seward \& Wang 1988). There are several methods to estimate the magnetic field in the synchrotron nebula (Ginzburg \& Syrovatskii 1965). All make use of the results from the spectral and spatial analysis (for a determination of the plerion size).

Limiting factors on the computations mentioned above are the correct determination of the size of the nebula, and a better derivation of parameters from the spectral analysis, both of which were only possible with Chandra. We present here the results of our analysis of our AO3 Chandra observation of MSH 11-62.

\section{First Part of the Puzzle: Existing Observations of MSH 11-62}

Radio observations of MSH 11-62 reveal a centrally brightened morphology with a Crab-like spectral index $\alpha$ of $0.29\left(S_{\nu} \propto \nu^{-\alpha}\right)$ and strong linear polarization (Roger et al. 1986; Whiteoak \& Green 1996). Einstein IPC (Wilson 1986) data were insufficient to identify the nature of the emission. We observed MSH 11-62 with $A S C A$ and showed that the remnant presents strong evidence for a central engine powering a synchrotron nebula (Harrus, Hughes \& Slane 1998). At energies below $2 \mathrm{keV}$, the emission is extended, covering a region with a diameter of somewhat more than $10^{\prime}$. At high energies, the extended emission which fills the radio shell has disappeared and only centrally concentrated emission remains.

The central hard emission detected at high energy is dominated by a nonthermal, power-law contribution with a photon index of $2.0_{-0.3}^{+0.1}$. The size of the hard X-ray source is consistent with an unresolved source $\left(<3^{\prime}\right)$. A second thermal contribution at a temperature of about $0.8 \mathrm{keV}$ contributes about $50 \%$ of the flux below $2 \mathrm{keV}$ (and less than $7 \%$ above).

\section{The End of the Puzzle? Chandra Observations of MSH 11-62}

We present here the results of the analysis of our $50 \mathrm{ks}$ Chandra observation of the region around the central source. The analysis on the rest of the remnant and our timing analysis of the XMM-Newton data $(35 \mathrm{ks})$ is being written up in a different paper. For the Chandra analysis, the data is corrected for CTI effects, screened for hot pixels and image corrected with a sub-pixel correction. 


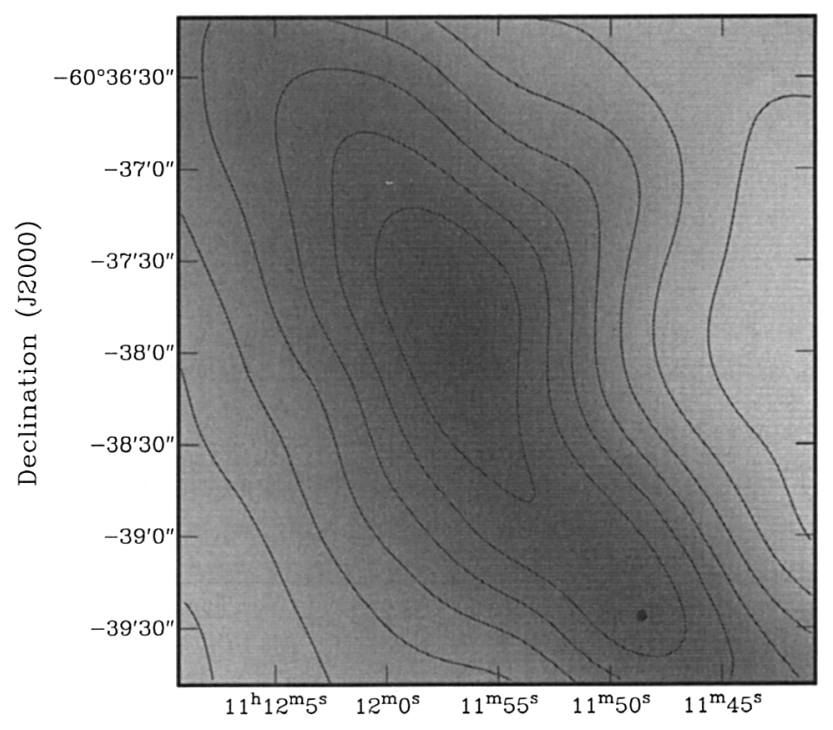

Right Ascension (J2000)

Figure 1. Image and contours from the ATCA at $1.4 \mathrm{GHz}$. The black dot is derived from the Chandra image at energies above $4 \mathrm{keV}$.

Figure 1 shows that the region of non-thermal emission is located at the tip of the central region of the radio emission as measured by the ATCA. We define a circular region around the maximum of the high-energy emission $(>4 \mathrm{keV})$ at $3 \sigma$ above background level. This translates to a circular region centered at $11^{\mathrm{h}} 11^{\mathrm{m}} 48 \mathrm{~s} .617,-60^{\circ} 39^{\prime} 26^{\prime \prime} \cdot 71(\mathrm{~J} 2000)$ and a radius of about $2^{\prime \prime}$. The background is modeled first as thermal-dominated emission from another region on the CCD. We then use this model (up to a free normalization) in our fit of the central region.

We find that the central source is best described by a power-law model with photon index $1.4 \pm 0.3$ and column density $N_{\mathrm{H}}$ of $\left(1.4_{-0.5}^{+0.4}\right) \times 10^{22}$ atoms $\mathrm{cm}^{-2}$. The unabsorbed flux of the non-thermal component from the central source is $\left(0.83_{-0.28}^{+0.44}\right) \times 10^{-13} \mathrm{ergs} \mathrm{cm}^{-2} \mathrm{~s}^{-1}$ below $2 \mathrm{keV}$ and $\left(2.7_{-1.5}^{+3.5}\right) \times 10^{-13} \mathrm{ergs} \mathrm{cm}^{-2} \mathrm{~s}^{-1}$ between 2 and $10 \mathrm{keV}$. The thermal component accounts for $59 \%$ of the total emission below $2 \mathrm{keV}$ and less than $2 \%$ above. A spectrum of the central region is shown in Figure 2. From the unabsorbed non-thermal flux between 0.2 and 4 $\mathrm{keV}$, we use an empirical relation between that value and the rotational energy loss $\dot{E}$ (Seward \& Wang 1988) to estimate an $\dot{E}$ between 3.7 and $7.6 \times 10^{36}$ ergs $\mathrm{s}^{-1}$ (at a distance of $3.5 \mathrm{kpc}$ ).

We also used the spectral slope found for MSH 11-62 to estimate its spindown energy loss rate using the relationship between the two derived from similar 


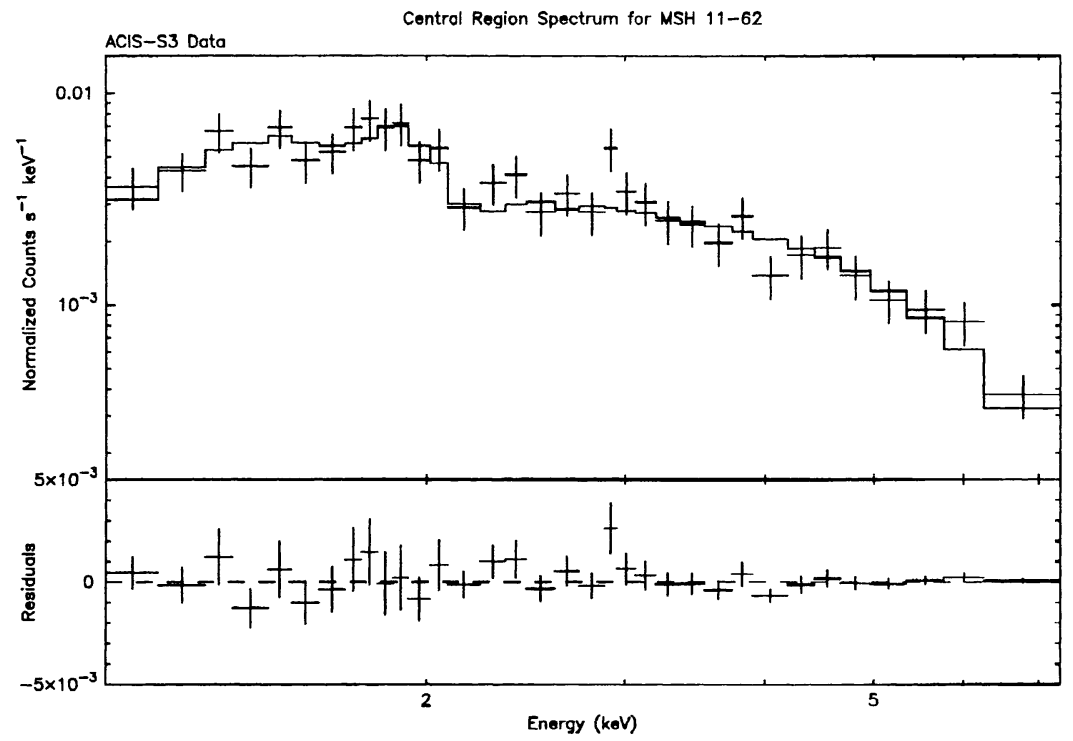

Figure 2. Best fit of the Chandra spectrum from the central region.

rotation-powered pulsars by Gotthelf (2003). We find an $\dot{E}$ between 0.7 and 3.3 $\times 10^{36} \mathrm{ergs} \mathrm{s}^{-1}$. We have also extracted the equivalent of the PWN using a region defined as $3 \sigma$ above background (computed on the total energy band and excluding the central so-called pulsar region). This encloses a region of about $5^{\prime \prime}$. We find that with a $N_{\mathrm{H}}$ fixed to the value derived from the analysis of the entire remnant, and if the emission from this region is described by a power law, its index is between 0.7 and 1.6. This is consistent with the values of $\Gamma_{\mathrm{PWN}}$ predicted from $\Gamma_{\mathrm{PSR}}$ of known young pulsars compiled in Gotthelf (2003).

Acknowledgments. This work was funded by grant number NRA 00-OSS07/03500279. The Australia Telescope is funded by the Commonwealth of Australia for operation as a National Facility managed by CSIRO. The author thanks Eric Gotthelf for his help.

\section{References}

Ginzburg, V. L., \& Syrovatskii, S. I. 1965, ARA\&A, 3, 297

Gotthelf, E. V. 2003, ApJ, 591, 361

Harrus, I. M., Hughes, J. P., \& Slane, P. O. 1998, ApJ, 499, 273

Roger, R., Milne, D. K., Caswell, J. L., \& Little, A. G. 1986, MNRAS, 219, 815

Seward, F. D., \& Wang, Z.-R. 1988, ApJ, 332, 199

Whiteoak, J. B. Z., \& Green, A. J. 1996, A\&AS, 118, 329

Wilson, A. S. 1986, ApJ, 302, 718 